\title{
Anticoagulation in cirrhosis: a new paradigm?
}

\author{
Filippo Leonardi, Nicola De Maria, and Erica Villa \\ Department of Internal Medicine, Gastroenterology Unit, Azienda Ospedaliero-Universitaria Policlinico di Modena, Modena, Italy
}

The liver plays a crucial role in coagulation cascade. Global hemostatic process is profoundly influenced by the presence of liver disease and its complications. Patients with cirrhosis have impaired synthesis of most of the factors involved in coagulation and fibrinolysis process due to a reduced liver function and altered platelet count secondary to portal hypertension. Altered routine tests and thrombocytopenia were considered in the past as associated with increased risk of bleeding. These concepts explain both the routine use of plasma and/or platelets transfusion in patients with liver cirrhosis, especially before invasive procedures, and why these patients were considered "auto-anticoagulated". New recent evidences show that patients with liver cirrhosis have a more complex hemostatic alteration. Despite the presence of altered levels of factors involved in primary hemostasis, coagulation and fibrinolysis, patients with stable cirrhosis have a rebalanced hemostatic, which however can easily be altered by decompensation or infection, both in hemorrhagic or thrombotic direction. Patients with cirrhosis have an increased risk of venous thrombotic events (namely portal vein thrombosis) while bleeding seems to be related to the grade of portal hypertension rather than to a hemostatic imbalance. The use of anticoagulants both as treatment or prophylaxis is safe, reduces the rate of portal vein thrombosis and decompensation, and improves survival. Standard laboratory coagulation tests are unable to predict bleeding and are inadequate for the assessment of hemostatic status in these patients, hence more comprehensive tests are required to guide the management of thrombotic and bleeding complications. (Clin Mol Hepatol 2017;23:13-21)

Keywords: Hemostasis; Cirrhosis; Anticoagulation

\section{INTRODUCTION}

Hemostasis is a complex physiological process aimed at stopping bleeding at the site of an injured blood vessel. The coagulation cascade integrates both cellular and humoral reactions driving in opposite directions, one leading toward blood clot formation, the other to activation of the anticoagulation system that controls the extension of the clot in order to avoid damage to the organs. 'The pro-coagulant factors, through the activation of the tissue factor by factor VII and its interaction with the platelets membranes, lead to thrombin generation, conversion from fibrino- gen to fibrin, and finally to blood clot formation. Meanwhile, thrombin itself, once complexed with its endothelial receptor thrombomodulin, activates plasma protein C. Activated protein C is a potent anticoagulant that, in combination with its plasma cofactor protein $S$, down-regulates thrombin generation through the inhibition of the activated forms of factors VIII and V. The anticoagulation effect is enhanced by plasma antithrombin, which, together with endothelial heparin-like substances, inhibits thrombin directly and indirectly through the inhibition of the activated forms of factors XI, IX, and X. Thrombin formation is also downregulated by the tissue factor pathway inhibitor (TFPI) that inhibits

\footnotetext{
Abbreviations:

EBL, endoscopic band ligation; INR, international normalized ratio; LMWH, low molecular weight heparins; PTT, partial thromboplastin time; PVT, portal vein thrombosis; TAFI, thrombin activatable fibrinolysis inhibitor; TF, tissue factor; TFPI, tissue factor pathway inhibitor; TIPS, transjugular intrahepatic porto-systemic shunt; tPA, tissue plasminogen activator; UPA, urokinase plasminogen activator; VTE, venous thromboembolism
}

\section{Corresponding author : Erica Villa}

Department of Internal Medicine, Gastroenterology Unit, Università degli Studi di Modena \& Reggio Emilia, and Azienda Ospedaliero-Universitaria di Modena, Via del Pozzo 71, Modena 41124, Italy

Tel: +390594225308, Fax: +390594222624

Email: erica.villa@unimore.it 
the complex tissue factor-factor VII and activated factor X. ${ }^{1}$ Under normal conditions, meaning in normal individuals, there are higher levels of pro- and anticoagulant proteins than are needed for minimal hemostatic function. This functional 'excess' allows for a high degree of stability: the hemostatic balance tends to be maintained even under stress.

\section{MODERN VIEW OF HEMOSTASIS: THE “CELL- BASED MODEL"}

In recent years, the "cell-based model" of hemostasis replaced the traditional "sequential model" and allowed a more accurate explanation of hemostatic process in vivo. ${ }^{2}$ Furthermore, in this model, it is possible to identify three different phases that simultaneously cooperate in vivo for adequate hemostasis: primary hemostasis, coagulation and fibrinolysis.

Primary hemostasis is defined as the interaction of platelets with exposed sub-endothelial surface at sites of vascular injury. Platelet adherence to sub-endothelium leads to the formation of the "platelet plug". This process is mediated by adherence of plasma proteins [most important the glycoprotein Von Willebrand Factor] to specific receptors exposed on platelets membranes, resulting in the adhesion of platelets to extracellular matrix proteins and subsequent aggregation to one another. A normal platelet number and function and normal plasma Von Willebrand Factor levels are all irreplaceable factors for a physiologic primary hemostasis.

Coagulation (or Secondary hemostasis) is defined as the formation of insoluble cross-linked fibrin by activated coagulation factors. It is initiated simultaneously by the exposure of tissue factor (TF) on sub-endothelial layer. Formation of factor VIla-TF complex initiate the coagulation cascade with activation of factor $X$ and factor IX. Activated factor $X$ and its cofactor (namely factor $V$ ) catalyze the activation of prothrombin to thrombin. At the same time, activated factor IX interacts with activated factor VIII, which is present on activated platelets membrane at site of vascular injury, and this complex activates factor $X$ with a strong amplification of thrombin generation. Coagulation may therefore be defined as the process that leads to thrombin generation through dynamic interaction of plasmatic coagulation proteins. Thrombin cleaves soluble fibrinogen causing the formation of insoluble fibrin clots, stabilized by activated factor XIII. In normal conditions thrombin generation is regulated by an anticoagulant system, including antithrombin, protein C (activated by interaction between thrombin and its receptor thrombomodulin), protein $S$ and the tissue factor pathway inhibitor (TFPI). The balance between these opposite systems prevents pathological thrombin generation and excessive clot formation as well as uncontrolled bleeding.

Fibrin deposition activates also a fibrinolytic system, a further control for unwanted clot formation. Principal effector of fibrinolysis is plasmin, a protease derived from plasminogen, which is regulated by two activators, tissue plasminogen activator (tPA) and urokinase plasminogen activator (UPA). Plasmin cleaves fibrin in soluble degradation products. Anti-activators also regulate the system with anti-fibrinolytic activity, including specific inhibitors of tPA (PAI-1), plasmin inhibitors (e.g. alpha-1 plasmin inhibitor) and thrombin activatable fibrinolysis inhibitor (TAFI). The balance between pro- and anti-fibrinolytic factors is necessary to prevent uncontrolled plasmin generation and hyper- or hypo-fibrinolysis. ${ }^{3}$

\section{ABNORMALITIES OF COAGULATION IN CHRONIC LIVER DISEASE}

The liver is fundamental in the architecture of hemostasis not only because it synthetizes the majority of the pro- and anticoagulants factors but also for the role played in their regulation, e. $\mathrm{g}$. in clearing these factors from circulation through its reticulo-endothelial system.

Liver disease profoundly affects coagulation. With the exception of Von Willebrand factor, which is secreted by endothelial cells, and calcium, it produces all factors involved in the coagulation process. Their production is impaired in case of hepatic insufficiency: this impairment is reflected by an increase in prothrombin time, which is the expression of the activity of factors I, II, V, VII and $X$. Thus, individuals with advanced liver disease were previously considered as having a hypocoagulant and prohemorrhagic condition, exacerbated by a reduction in platelets count (due to portal hypertension). ${ }^{4}$ As a consequence, patients with liver cirrhosis were supposed to be protected against thrombosis. However, this belief is not correct: as patients with cirrhosis also have a reduction in anticoagulant proteins, to include antithrombin III, protein $\mathrm{S}$, or $\mathrm{C}$, and an increase of procoagulant factors (such as factor VIII or von Willebrand factor) their coagulative situation is characterized by a new balance of pro- and anticoagulation factors, defined as "rebalanced hemostasis", with an inclination toward bleeding or thrombotic events depending on the clinical settings. The hemostatic balance is much harder to maintain when the levels of the pro- and anticoagulant factors are reduced by 
Filippo Leonardi, et al.

hepatic insufficiency, but still there may not be a tendency to hemorrhage or thrombosis. However, in the occurrence of stressful conditions, like infections, encephalopathy or bleeding, the fragile coagulative balance may be disrupted.

Fibrinolytic system is also conditioned by hepatic function, as plasminogen, antiplasmin (alpha-2 plasmin inhibitor), plasmin inhibitor and TAFI are synthetized in the liver. Furthermore, in liver disease plasma levels of both TPA and its inhibitor PAI-1 (due to endothelial activation and reduced hepatic clearance) are increased. $^{5}$

Finally, chronic liver disease is usually characterized by a reduction of platelet count and alteration in platelet function. Thrombocytopenia is the result of increased splenic and hepatic sequestration and destruction due to portal hypertension, as well as to reduced hepatic synthesis of thrombopoietin, a hormone that regulate proliferation and differentiation of megakaryocytes and platelet formation. Thrombocytopathy results from dysfunction of platelets due to alteration of proteins involved in platelet aggregation process, including defective thromboxane A-2 synthesis by activated platelets or altered glycoprotein $1 \mathrm{~b}$, a platelet's surface membrane protein.

In summary, the decreased hepatic synthetic function and the hemodynamic alterations due to portal hypertension exert a profound influence on the hemostatic balance, accounting for the presence of altered coagulative parameters in patients with chronic liver disease. Routine blood tests (like International Normalized Ratio [INR] or Partial Thromboplastin Time [PTT]) do not accurately evaluate the coagulative performance in these patients. When more dynamic tests like thromboelastography are used, the apparently deranged hemostasis is much less affected and is not associated with relevant complications even in invasive procedures. $^{6}$

\section{THROMBOTIC COMPLICATIONS IN CIRRHOSIS}

As we had shown in the previous paragraph, recent evidences indicate that patients with liver disease are not more likely to develop a hemorrhagic complication rather than a thrombotic one. Concepts like "auto-anticoagulation" or "natural protection of cirrhotic patients against thrombotic events" are outdated. Increased occurrence instead of venous thromboembolism (VTE) or venous thrombotic events (Portal Vein Thrombosis, PVT) has been recently reported.

\section{Venous thromboembolism (VTE)}

Several studies indicate the incidence of non-portal VTE in patients with chronic liver disease in a range between $0.5 \%$ and $6.3 \% .^{7-9}$ Two retrospective studies by Northup et al. ${ }^{7}$ and GarciaFuster et al. ${ }^{8}$, showed that the risk of developing VTE increases in patients with low serum albumin, but is independent from elevated INR or low platelet count. Serum albumin is a marker of liver synthetic function, and hence, indirectly, of the imbalance of serum levels of anticoagulant factors as antithrombin III, protein $C$ and protein $\mathrm{S}$ : this explains the increased risk of VTE in these patients. A retrospective study by Dabbagh et al. ${ }^{9}$ confirmed that an elevated INR (even >2.2) does not protect patients with chronic liver disease against the risk of VTE. Other studies showed that not only patients with cirrhosis are not naturally auto-anticoagulated, but also they seem to have an increased relative risk of venous thromboembolism (both deep vein thrombosis and pulmonary embolism) compared with controls. ${ }^{9-11}$ Wu et al. ${ }^{12}$ assessed the incidence of VTE in patients with compensated or decompensated cirrhosis and a random sample of control patients. Interestingly, results indicate that patients younger than 45 years have an increased risk of VTE independently from being compensated or decompensated. After 45 years of age, patients with compensated cirrhosis had a lower risk of VTE, while those with decompensated disease had a similar risk compared with controls. These results may be explained with age-related factors not correlated with liver disease; in younger patients, who normally have low baseline risk of VTE, presence of cirrhosis imply an increased risk of VTE compared with non cirrhotic patients, whereas in older patients extrahepatic factors seem to outweigh liver-related risk factors.

\section{Portal vein thrombosis}

Portal vein thrombosis (PVT), the obstruction of portal vein and/or tributaries, is a relatively frequent event in cirrhotic patients; its incidence varying from $7.4 \%$ to $17.9 \%$ in different studies (Table 1). ${ }^{13-15}$ The development of PVT in cirrhosis is directly proportional to disease severity, being more frequent in individuals with decompensated cirrhosis. Although severe portal hypertension together with a reduction in portal blood flow speed facilitates the development of splanchnic venous thrombosis in patients with cirrhosis, the derangement of hemostatic balance in ALT plays also an important role especially in stressful clinical conditions. Beside decompensation, other situations are known risk factors for PVT in patients with cir- 
Table 1. Prevalence of PVT in cirrhosis in different clinical settings

\begin{tabular}{lc}
\hline Type of PVT & $\%$ \\
\hline Overt & 0.6 \\
\hline Screening for HCC & 7.0 \\
\hline In-Hospital & $8.0-40$ \\
\hline Necropsy & $15.0-25$ \\
\hline Before LTx or PSS & \\
Unrecognized & 64 \\
\hline Small mural thrombus & 25 \\
\hline Large veins (intimal fibrosis) & 36 \\
\hline Small veins (intimal fibrosis) & \\
\hline PVT, portal vein thrombosis; HCC, hepatocellular carcinoma; LTx, liver \\
transplantation; PSS, portosystemic shunt.
\end{tabular}

rhosis, to include endoscopic treatment of varices, abdominal surgery, injury to the portal venous system, and paradoxically also low platelets count, as well as bacterial infections. All these events are able to trigger the intrinsic pathway of coagulation leading to blood clot formation.

The pathogenesis of PVT includes both "systemic factors", like those mentioned previously (coagulation abnormalities or presence of antiphospholipid antibodies ${ }^{16}$ ), and "local factors", as peri-portal lymphangitis and fibrosis that lead to alteration of liver cytoarchitecture with consequent flow reduction and endothelial activation. ${ }^{17-19}$ Clinical presentation of PVT varies from asymptomatic to a medical emergency, depending on the extension and the rapidity of thrombus formation: "acute PVT" may be characterized by intestinal congestion and ischemia to peritonitis, shock and death. ${ }^{20}$ "Chronic PVT" may be asymptomatic, but when discovered, the presence of varices and ascites should be investigated. Ultrasonography and Doppler ultrasound are usually first-line imaging method for the diagnosis of PVT, with a sensitivity and specificity variable from $66 \%$ to 100\%. ${ }^{21}$ Computed Tomography and Magnetic Resonance Imaging are however better for determining presence and extent of thrombosis, with a sensitivity and specificity near $100 \%$. $22-24$

\section{ANTICOAGULATION IN CIRRHOSIS}

\section{Treatment of established PVT}

Management of PVT in patients with cirrhosis is not reported in any guideline. There are no definitive consensus publications for prevention, treatment and monitoring of PVT in these pa- tients. Anticoagulation is usually the first-line therapy, allowing a good chance of complete recanalization, a reduced incidence of portal hypertension complications and a decreased rate of thrombosis progression. ${ }^{24}$ Several studies are available indicating that anticoagulation, either through low molecular weight heparins (LMWH) or vitamin $\mathrm{K}$ antagonists' administration, is both safe and effective in treating PVT (Table 2). ${ }^{12,24-36}$ According to the published data, following anticoagulation the rate of portal vein repermeation ranges from 40 to $>90 \%$, as compared to $0 \%$ of the untreated controls. Severe side effects are practically absent, very few occurring with vitamin $\mathrm{K}$ antagonists. Importantly, increased hemorrhagic events are not reported, and chronic administration of LMWH does not affect the outcome of gastro-intestinal bleeding following varices rupture: in a retrospective multicentric trial published in 2015, anticoagulation treatment for PVT or cardiovascular disorders did not increase neither the rate of treatment failure following varices rupture nor the 6 months mortality rate. ${ }^{37}$ Initiation of anticoagulation as early as possible is recommended, because early initiation of anticoagulation is the only factor significantly associated with repermeation. Anticoagulation should be initiated with subcutaneous LMWH, which is as effective as intravenous heparin, does not require laboratory monitoring, has a more predictable dose response relationship, and has a lower risk of adverse events (i.e., heparin-induced thrombocytopenia). Anticoagulation is to be maintained for at least a 6 month duration, as repermeation beyond 4-6 months is unlikely; unfortunately, the recurrence of thrombosis after complete repermeation occurs in more than $35 \%$ of patients after stopping anticoagulation. Lifelong anticoagulation should be decided on an individualized case-to-case basis.

It is advisable, before starting anticoagulation, to evaluate the presence of varices, and, in case of large varices or previous history of gastro-intestinal bleeding, start prophylaxis, either with beta-blockers or endoscopic band ligation. Transjugular Intrahepatic Porto-systemic Shunt (TIPS) results a valid alternative when anticoagulation fails, with a reported complete recanalization of portal venous system in $57 \%$ of patients with cirrhosis, and a decrease in thrombosis in $30 \%$ of patients, but with the limitation that it could be considered only in experienced centers. ${ }^{38}$

\section{Prophylaxis of PVT}

Since portal thrombosis is to be avoided, efforts should be 
Filippo Leonardi, et al.

Anticoagulation in cirrhosis

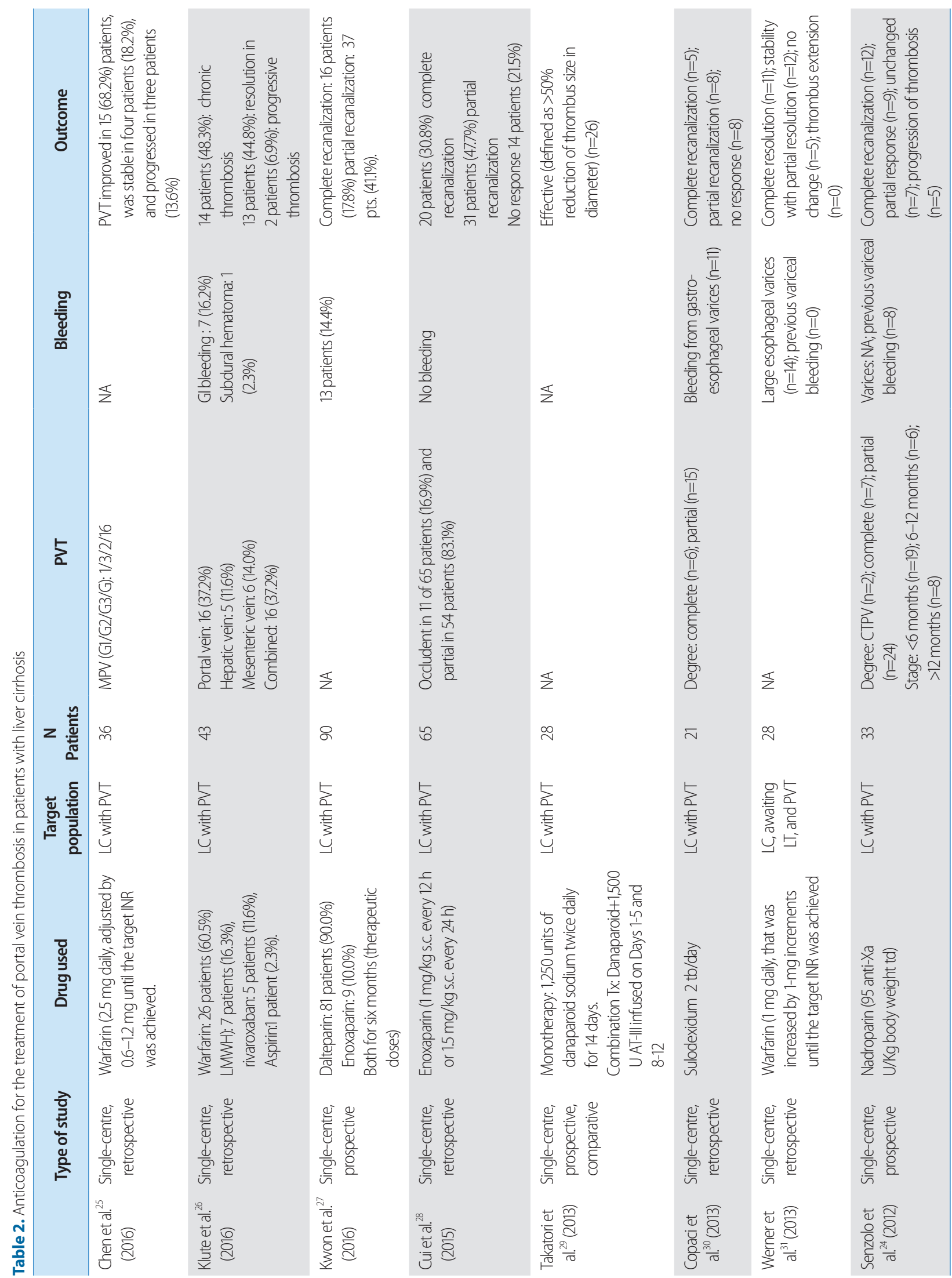




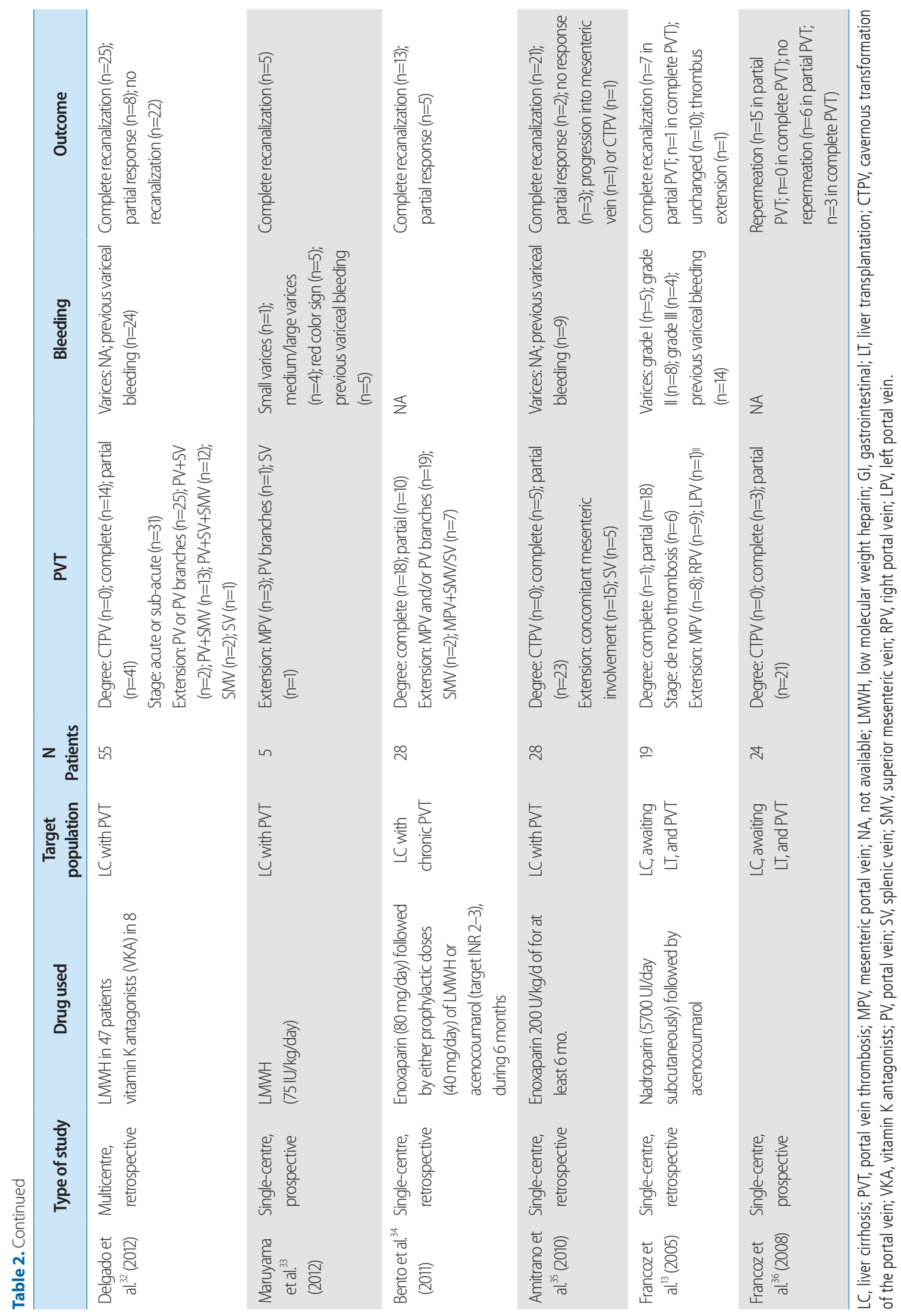


Filippo Leonardi, et al.

made also to prevent its development in individuals with cirrhosis. However, the use of anticoagulation in subjects with end-stage liver disease raised in the past some concerns about its safety, because heparin or vitamin $\mathrm{K}$ antagonists may tip the unsteady hemostatic balance of patients with cirrhosis toward bleeding. These concerns were probably excessive.

In 2012 we published a controlled trial where individuals with advanced cirrhosis were randomly assigned to receive or not enoxaparin 4,000 U subcutaneously daily: after 96 weeks, no PVT was seen in the treated group while $36 \%$ of the subjects in the control group had signs of portal thrombosis. Moreover, no relevant side effects or hemorrhagic events were reported in the treated group, which showed also a lower incidence of hepatic decompensation and an improved overall survival as compared to the untreated group. Prevention of PVT may not be the only event responsible for all these beneficial effects: heparin treatment itself could be of advantage in preventing the progression of liver disease. A possible mechanism is that anticoagulation improves intestinal microcirculation, which in turn reduces bacterial translocation and thus the risk of bacterial infections.

So far, this is the only published study on prophylaxis. However, two other multicenter randomized controlled study were started: one is Childbenox (NCT02271295) and the other is Cirroxaban (NCT02643212). While Childbenox protocol is very similar to our protocol and therefore it will allow, once completed, a meta-analysis of the raw data, Cirroxaban uses the new oral anticoagulant Rivaroxaban. Both are expected to provide data on the risks and benefits of proposing anticoagulation in order to prevent PVT; most importantly, they will be able to confirm or not the beneficial effect on decompensation and survival shown in our study.

\section{Safety of anticoagulation}

Treatment with LMWH was safe and effective, and not related with an increased occurrence of bleeding. A recent retrospective analysis evaluating overall gastrointestinal bleeding in cirrhotic patients treated with anticoagulants ruled out anticoagulation as an independent risk factor for bleeding or death. ${ }^{36}$ A confirmation to these results was found in a retrospective cohort of patients with liver cirrhosis undergoing elective Endoscopic Band Ligation (EBL). Among 26,742 upper endoscopies performed during the study period, 542 were prophylactic EBLs performed on 245 (200 primary and 45 secondary pro- phylaxis) patients with liver cirrhosis. $197 \mathrm{EBL}$ sessions were performed on 76 cases $(4.81 \pm 0.92$ bands per session) who were on LMWH while $345 \mathrm{EBL}$ sessions were performed in 169 controls $(4.80 \pm 1.03)$. Clinical and endoscopic features were similar between the groups at the time of the first EBL. Overall 5 patients bled (2\%), 3 cases (3.9\%) and 2 controls (1.2\%, log rank 0.161$)$, thus further confirming that anticoagulation with LMWH does not increase the risk of post-procedural bleeding patients with liver cirrhosis undergoing prophylactic EBL. ${ }^{39}$

\section{CONCLUSIONS AND FUTURE DIRECTIONS}

Patients with liver cirrhosis have a more complex coagulation state than previously believed. They display a global hemostatic alteration, involving both pro- and anticoagulant factors. However, compensated patients have a substantially normal coagulative performance ("rebalanced hemostasis").,4,40 Decompensated patients present bleeding as a complication of portal hypertension, mostly from gastro-intestinal varices. However, the contribution of deranged hemostasis as a precipitating factor is poorly documented and the impact of coagulation in this setting is not well established. What it is clear is that standard coagulation tests are inappropriate to define the complex coagulation state of patients with liver cirrhosis and dynamic tests like thromboelastography should be used instead. $^{6}$

The occurrence of thrombotic events, which are major contributors of variceal bleeding, has been previously underestimated. A better knowledge of this complication and of its mechanisms has changed the paradigm and the clinical management of the patients (i.e. the use of anticoagulants). Anticoagulation should be started as soon as the thrombotic complication is diagnosed and continued for at least 6 months. There are no strict indications on whether it should be continued indefinitely.

The major challenge, however, is to define whether prophylactic anticoagulation should be instituted in patients with cirrhosis and when. Data from a prospective randomized study ${ }^{14}$ showed that anticoagulation not only prevented the occurrence of portal vein thrombosis but, most importantly, decreased the occurrence of decompensation and improved survival. Two other studies with anticoagulants and with the same endpoints are currently ongoing (Childbenox, NCT02271295, and Cirroxaban, NCT02643212). Their results are eagerly awaited. 


\section{Conflicts of Interest}

The authors have no conflicts to disclose.

\section{REFERENCES}

1. Tripodi A, Primignani $M$, Chantarangkul V, Dell'Era A, Clerici $M$, de Franchis $R$, et al. An imbalance of pro- vs anticoagulation factors in plasma from patients with cirrhosis. Gastroenterology 2009;137:2105-2111.

2. Hoffman M, Monroe DM 3rd. A cell-based model of hemostasis. Thromb Haemost 2001;85:958-965.

3. Hoffman M, Monroe DM. Coagulation 2006: a modern view of hemostasis. Hematol Oncol Clin North Am 2007;21:1-11.

4. Tripodi A, Mannucci PM. The coagulopathy of chronic liver disease. N Engl J Med 2011;365:147-156.

5. Leebeek FW, Kluft C, Knot EA, de Maat MP, Wilson JH. A shift in balance between profibrinolytic and antifibrinolytic factors causes enhanced fibrinolysis in cirrhosis. Gastroenterology 1991;101:13821390.

6. De Pietri L, Bianchini M, Montalti R, De Maria N, Di Maira T, Begliomini $B$, et al. Thrombelastography-guided blood product use before invasive procedures in cirrhosis with severe coagulopathy: $A$ randomized, controlled trial. Hepatology 2016;63:566-573.

7. Northup PG, McMahon MM, Ruhl AP, Altschuler SE, Volk-Bednarz A, Caldwell $\mathrm{SH}$, et al. Coagulopathy does not fully protect hospitalized cirrhosis patients from peripheral venous thromboembolism. Am J Gastroenterol 2006;101:1524-1528.

8. García-Fuster MJ, Abdilla N, Fabiá MJ, Fernández C, Oliver V, Forner M J. [Venous thromboembolism and liver cirrhosis]. Rev Esp Enferm Dig 2008;100:259-262.

9. Dabbagh O, Oza A, Prakash S, Sunna R, Saettele TM. Coagulopathy does not protect against venous thromboembolism in hospitalized patients with chronic liver disease. Chest 2010;137:1145-1149.

10. Gulley D, Teal E, Suvannasankha A, Chalasani N, Liangpunsakul S. Deep vein thrombosis and pulmonary embolism in cirrhosis patients. Dig Dis Sci 2008;53:3012-3017.

11. Søgaard KK, Horváth-Puhó E, Grønbaek H, Jepsen P, Vilstrup $H$, Sørensen HT. Risk of venous thromboembolism in patients with liver disease: a nationwide population-based case-control study. Am J Gastroenterol 2009;104:96-101.

12. Wu H, Nguyen GC. Liver cirrhosis is associated with venous thromboembolism among hospitalized patients in a nationwide US study. Clin Gastroenterol Hepatol 2010;8:800-805.

13. Francoz C, Belghiti J, Vilgrain V, Sommacale D, Paradis V, Condat B, et al. Splanchnic vein thrombosis in candidates for liver transplantation: usefulness of screening and anticoagulation. Gut 2005;54:691697.
14. Villa E, Cammà C, Marietta M, Luongo M, Critelli R, Colopi S, et al. Enoxaparin prevents portal vein thrombosis and liver decompensation in patients with advanced cirrhosis. Gastroenterology 2012;143:1253-1260.

15. Abdel-Razik A, Mousa N, Elhelaly R, Tawfik A. De-novo portal vein thrombosis in liver cirrhosis: risk factors and correlation with the Model for End-stage Liver Disease scoring system. Eur J Gastroenterol Hepatol 2015;27:585-592.

16. Violi F, Ferro D, Basili S, D'Angelo A, Mazzola G, Quintarelli C, et al. Relation between lupus anticoagulant and splanchnic venous thrombosis in cirrhosis of the liver. BMJ 1994;309:239-240.

17. Wang J, Zhao H, Liu Y. Portal vein thrombosis. Hepatobiliary Pancreat Dis Int 2005;4:515-518.

18. Zocco MA, Di Stasio E, De Cristofaro R, Novi M, Ainora ME, Ponziani $F$, et al. Thrombotic risk factors in patients with liver cirrhosis: correlation with MELD scoring system and portal vein thrombosis development. J Hepatol 2009;51:682-689.

19. Hoekstra J, Janssen HL. Vascular liver disorders (II): portal vein thrombosis. Neth J Med 2009;67:46-53.

20. Condat B, Valla D. Nonmalignant portal vein thrombosis in adults. Nat Clin Pract Gastroenterol Hepatol 2006;3:505-515.

21. Ueno N, Sasaki A, Tomiyama T, Tano S, Kimura K. Color Doppler ultrasonography in the diagnosis of cavernous transformation of portal vein. J Clin Ultrasound 1997;25:227-233.

22. Bach AM, Hann LE, Brown KT, Getrajdman GI, Herman SK, Fong Y, et al. Portal vein evaluation with US: comparison to angiography combined with CT arterial portography. Radiology 1996;201:149-154.

23. Kreft B, Strunk H, Flacke S, Wolff M, Conrad R, Gieseke J, et al. Detection of thrombosis in the portal venous system: comparison of contrast-enhanced MR angiography with intraarterial digital subtraction angiography. Radiology 2000;216:86-92.

24. Senzolo M, M Sartori T, Rossetto V, Burra P, Cillo U, Boccagni P, et al. Prospective evaluation of anticoagulation and transjugular intrahepatic portosystemic shunt for the management of portal vein thrombosis in cirrhosis. Liver Int 2012;32:919-927.

25. Chen H, Liu L, Qi X, He C, Wu F, Fan D, et al. Efficacy and safety of anticoagulation in more advanced portal vein thrombosis in patients with liver cirrhosis. Eur J Gastroenterol Hepatol 2016;28:82-89.

26. Klute K, Defilippis EM, Shillingford K, Chapin J, DeSancho MT. Clinical presentations, risk factors, treatment and outcomes in patients with splanchnic vein thrombosis: a single-center experience. J Thromb Thrombolysis 2016;42:267-271.

27. Kwon JH, Koh YG, Yoon JH, Yu SJ. Anticoagulation treatment with low molecular-weight heparin for portal vein thrombosis in liver cirrhosis: Efficacy and risk of hemorrhagic complications. Blood 2016;128:1435.

28. Cui SB, Shu RH, Yan SP, Wu H, Chen Y, Wang L, et al. Efficacy and safety of anticoagulation therapy with different doses of enoxaparin 
Filippo Leonardi, et al. Anticoagulation in cirrhosis

for portal vein thrombosis in cirrhotic patients with hepatitis B. Eur J Gastroenterol Hepatol 2015;27:914-919.

29. Takatori H, Hayashi T, Sunagozaka H, Arai K, Kitamura K, Kagaya T, et al. Danaparoid sodium monotherapy for portal vein thrombosis in cirrhotic patients is as effective as combination therapy with antithrombin III. [Abstract]. Hepatology 2013;58:894A.

30. Copaci I, Ismail G, Micu L, Ioanitescu S, Chiriac G. Anticoagulant therapy with sulodexidum for portal vein thrombosis in patients with liver cirrhosis [abstract]. Hepatology 2013;58:67A-868A.

31. Werner KT, Sando S, Carey EJ, Vargas HE, Byrne TJ, Douglas DD, et al. Portal vein thrombosis in patients with end stage liver disease awaiting liver transplantation: outcome of anticoagulation. Dig Dis Sci 2013;58:1776-1780.

32. Delgado MG, Seijo S, Yepes I, Achécar L, Catalina MV, García-Criado $A$, et al. Efficacy and safety of anticoagulation on patients with cirrhosis and portal vein thrombosis. Clin. Gastroenterol. Hepatol 2012;10:776-783.

33. Maruyama H, Takahashi M, Shimada T, Yokosuka O. Emergency anticoagulation treatment for cirrhosis patients with portal vein thrombosis and acute variceal bleeding. Scand J Gastroenterol 2012;47:686-691.

34. Bento L, Rodriguez Huerta A, Pascual C, Pérez Rus G, Catalina V, Yepes I, et al. Antithrombotic Therapy in Non-Neoplastic Chronic
Portal Venous Thrombosis in Cirrhosis: Recanalization and Liver Function Evaluation. Blood 2011;118:3358.

35. Amitrano L, Guardascione MA, Menchise A, Martino R, Scaglione M, Giovine $S$, et al. Safety and efficacy of anticoagulation therapy with low molecular weight heparin for portal vein thrombosis in patients with liver cirrhosis. J Clin Gastroenterol 2010;44:448-451.

36. Francoz C, Dondero F, Abdelrazek W, Valla D, Sommacale D, Belghiti J, et al. Screening for portal vein thrombosis in candidates for liver transplantation and anticoagulation until transplantation: Results of a prospective assessment. Liver Transpl 2008;14(Suppl 1):S245.

37. Cerini F, Gonzalez JM, Torres F, Puente Á, Casas M, Vinaixa C, et al. Impact of anticoagulation on upper-gastrointestinal bleeding in cirrhosis. A retrospective multicenter study. Hepatology 2015;62:575-583.

38. Luca A, Miraglia R, Caruso S, Milazzo M, Sapere C, Maruzzelli L, et al. Short- and long-term effects of the transjugular intrahepatic portosystemic shunt on portal vein thrombosis in patients with cirrhosis. Gut 2011;60:846-852.

39. Bianchini M, Cavani G, Bonacorso A, Turco L, Merighi A, Villa E, et al. Low Molecular Weight Heparin Treatment Does not Increase the Risk of Bleeding after Prophylactic Endoscopic Variceal Band Ligation in Patients with Cirrhosis. J Hepatol 2016;64(Suppl 2):S662-S663.

40. Lisman T, Porte RJ. Rebalanced hemostasis in patients with liver disease: evidence and clinical consequences. Blood 2010;116:878-885. 